\title{
Upper Limits for the Number of Bound States Associated with the Yukawa Potential
}

\author{
Herbert S. Bennett* \\ National Bureau of Standards, Washington, DC 20234
}

July 23,1980

\begin{abstract}
The number of bound-state solutions of the Schrödinger equation for the screened Coulomb potential (Yukawa potential), $-(C / r) \exp (-\alpha r)$, occurs frequently in theoretical discussions concerning, for example, gas discharges, nuclear physics, and semiconductor physics. The number of bound states is a function of $(C / \alpha)$. Three upper limits for the number of bound states associated with the Yukawa potential are evaluated and compared. These three limits are those given by Bargmann, Schwinger, and Lieb. In addition, the Sobolev inequality states that whenever $(C / \alpha)<1.65$ no bound state occurs. This agrees to within a few percent of the numerical calculations of Bonch-Bruevich and Glasko. The Bargmann and Lieb limits and the Sobolev inequality are substantially easier to evaluate than the Schwinger limit. Among the three limits, the Schwinger limit gives the most restrictive limit for the existence of only one bound state and, therefore, is the best one to use for the approach to no binding, i.e., $1.65<(C / \alpha) \leq 1.98$. The Lieb limit is the best among the three when $(C / \alpha)>1.98$. The Bargmann limit is the least restrictive.
\end{abstract}

Key words: Bargmann limits; bound-state estimates; Lieb limits; number of bound states; Schwinger limits; screened Coulomb potential; Sobolev's inequality; Yukawa potential.

\section{Introduction}

Many theoretical topics in such areas as plasma, nuclear, solid state, and semiconductor physics [1-6] ${ }^{1}$ require knowledge about the number of bound-state solutions of the Schrodinger equation for two particles that interact according to the Yukawa or screened Coulomb potential. For example, as semiconductor devices become smaller, required dopant ion densities and their spatial gradients increase. The density of states for such highly doped materials is needed to predict device performance and degradation mechanisms. The density of states involves summation over bound states and integration over continuum states. The disappearance of bound-impurity levels in semiconductors due to free-carrier screening of the Coulomb field of impurity ions is thereby fundamental in understanding the behavior of electronic devices. [7]

During the course of research on the effect of donor impurities on the continuum electronic states in semiconductors, the author has used the Bargmann limit [8], the Schwinger limit [9], and the Lieb limit [10] to evaluate upper limits for the number of bound states associated with the screened Coulomb potential. The analytic methods used here to calculate these limits are more general and may be applied to other fields such as those mentioned above.

We shall show that 1) when the Yukawa potential permits only one bound state to exist, Schwinger's extension of Bargmann's limit gives a more restrictive limit for the number of bound states than do the Bargmann and Lieb limits; 2) when many bound states exist, the Schwinger and Bargmann limits agree to within a few percent and are not as restrictive as the Lieb limit; and 3) the Sobolev inequality [10,11] predicts no binding when $(C / \alpha)<1.65$. The limit 1.65 from the Sobolev inequality agrees to within a few percent of the limit 1.68 from the numerical work of Bonch-Bruevich and Glasko. [5]

\footnotetext{
*Center for Electronics and Electrical Engineering, National Engineering Laboratory.

${ }^{1}$ Figures in brackets indicate literature references at the end of this paper.
} 


\section{Yukawa Potential and Schrödinger Equation}

The Schrödinger equation for two particles that interact according to the Yukawa potential is

$$
\left[\nabla^{2}+\frac{2 m}{\hbar^{2}}\{E-V(r)\}\right] \psi(r)=0,
$$

where $E$ is the energy of the two-particle system, $m$ is the reduced or effective mass, $\mathbf{r}$ is the relative coordinate vector between the two particles, and $\mathbf{r}=|\mathbf{r}|$ is its magnitude. The spherically symmetric potential energy $V(r)$ is assumed to have the Yukawa form:

$$
V(r)=-C r^{-1} \exp (-\alpha r)
$$

For many applications, the Yukawa potential is an idealized one which gives a reasonable description of the physical phenomena under study. Expressions for the parameters $C$ and $\alpha$ in terms of physical quantities then depend upon the application. For donors such as phosphorus or arsenic in silicon, the quantities $C$ and $\alpha$ become, respectively, $C=\left(e^{2} / \epsilon\right)$ and $\alpha=1 / r_{s}$. The charge of the electron is $e$, the dielectric constant is $\epsilon$, and the screening length is $r_{s}$. The last depends in part upon the temperature and the dopant density. It gives the extent to which free carriers screen the Coulomb field of the donor ion.

The radial wave equation obtained from the Schrödinger equation, eq. (1), for the spherically symmetric potential $V(r)$ is

$$
\frac{d^{2}}{d r^{2}} \phi_{\ell}(r)+\left[\frac{2 m}{\hbar^{2}}\{E-V(r)\}-\frac{\ell(\ell+1)}{r^{2}}\right] \phi(r)=0
$$

where

$$
\psi(\underset{\sim}{r})=\Sigma a_{\ell, m} \phi_{\ell}(r) Y_{\ell m}(\theta, \phi) .
$$

The quantities, $\Sigma_{l m}$, are constants. The normalization and orthogonality conditions for the spherical harmonics $Y_{m}(\Theta, \phi)$ are

$$
\int_{0}^{2 \pi} d \phi \quad \int_{0}^{\pi} d \theta \sin Y_{l_{1} m_{1}}(\theta, \phi) Y_{l_{2} m_{2}}(\theta, \phi)=\delta_{\ell_{1} \ell_{2}} \delta_{m_{1} m_{2}} .
$$

In the following sections, we shall calculate upper limits for the total number of bound-state solutions $N$ of the Schrödinger equation, equation (l), and the number of bound-state solutions $n_{\ell}$ of the radial wave equation, eq. (3), for a given $\mathrm{l}$. Counting multiplicity, we perform the following summation to obtain the total number of bound states for a given spherically symmetric potential $V$.

$$
N[V]=\sum_{\ell=0}^{I_{\max }}(2 \ell+1) \mathrm{n}_{\ell}[V]
$$

where $\ell_{\max }$ is the largest value of $\ell$ for which $n_{\ell} \neq 0$.

\section{Bargmann Limits}

When the integral,

$$
I_{B}[V]=\int_{0}^{\infty} r|V(r)| d r
$$

is finite, the Bargmann formulation [8] gives the inequality that

$$
(2 \ell+1) n_{\ell}<I_{B}[V] .
$$


Thus, for the Yukawa potential, eq. (2), we have

$$
I_{B}[V]=(C / \alpha)
$$

From the inequality (7), we note that the maximum $\ell=\ell_{\max }$ for which $n_{\ell} \neq 0$ in the sum of eq. (5) has the upper limit $1 / 2\left(I_{B}-1\right)$. Thus, we have the following inequality [9] for spherically symmetric potentials; namely,

$$
N<\frac{1}{2} I_{B}\left(I_{B}+1\right)
$$

\section{Schwinger Limits}

Schwinger [9] extends the Bargmann derivation to treat angular and spin-independent potentials and to give the number of states that lie at or below some chosen energy. His result for the upper bound $I_{s}$ to the total number of bound states $N$ associated with a general spin-independent three-dimensional potential $V(r)$ is

$$
N<I_{s}
$$

where

$$
I_{S}=\frac{1}{(4 \pi)^{2}} \iint d^{3} r d^{3} r^{\prime} \frac{\left.\mid V(\mathbf{r})\rfloor \mid V\left(\mathbf{r}^{\prime}\right)\right\rfloor}{|\mathbf{r}-\mathbf{r}|^{2}}
$$

We shall now proceed to evaluate the double integral in eq (11) for the case of the Yukawa potential given by eq. (2). Because the Yukawa potential is spherically symmetric, we expand the denominator in terms of the spherical harmonics; namely,

$$
\frac{1}{\left|\mathbf{r}-\mathbf{r}^{\prime}\right|}=4 \pi \sum_{\ell=0}^{\infty} \sum_{m=-\ell}^{\ell} \frac{1}{(2 \ell+1)} \frac{\mathrm{r}_{<}^{l}}{\mathrm{r}_{>}^{\ell+1}} Y_{\ell m}\left(\theta^{\prime}, \phi^{\prime}\right) Y_{l m}(\theta, \phi)
$$

where $r_{<}$and $r_{>}$are, respectively, the lesser and the greater values of $|\mathbf{r}|$ and $\left|\mathbf{r}^{\prime}\right|$ and where

$$
Y_{l m}(\theta, \phi)=\left\{\frac{(2 \ell+1)(\ell-m) !}{4 \pi(\ell+m) !}\right\}^{1 / 2} \mathrm{P}_{\ell}^{m}(\cos \theta) e^{i m \phi}
$$

The Legendre polynomials $P_{\ell}^{m}$ satisfy the relation

$$
P_{\ell}^{-m}(x)=(-1)^{m} \frac{(\ell-m) !}{(\ell+m) !} P_{\ell}^{m}(x) .
$$

Substituting relation (12) into eq. (11) and using for the volume element in spherical coordinates $d^{3} r=r^{2}$ $\sin \theta d \theta d \phi d r$, we perform the integrations over $\theta, \phi, \theta^{\prime}$, and $\phi^{\prime}$ by frequent reference to eqs. (4), (13), and (14) and obtain the result that for spherically symmetric potentials $V_{s}(r)$

$$
\begin{aligned}
I_{s}\left[V_{s}\right]= & \int_{0}^{\infty} r^{2} d r \int_{0}^{\infty} r^{\prime 2} d r^{\prime}\left|V_{s}(r)\right|\left|V_{s}\left(r^{\prime}\right)\right| \\
& \times \sum_{\ell=0}^{\infty} \frac{1}{(2 \ell+1)} \frac{r_{\ell}^{2 \ell}}{r_{>}^{2 \ell+2}} .
\end{aligned}
$$

Equation (15) becomes for the Yukawa potential (2)

$$
\begin{gathered}
I_{S}(\mathrm{Y})=(C / \alpha)^{2} \sum_{\ell=0}^{\infty} \frac{1}{(2 \ell+1)}\left[\int_{0}^{\infty} z \exp (-z) d z\right. \\
\left.\times\left\{\int_{0}^{1} \eta^{2 \ell+1} \exp (-z \eta) d \eta+\int_{1}^{\infty} \eta^{-2 l-1} \exp (-z \eta) d \eta\right]\right],
\end{gathered}
$$


where we made the substitutions $z=\alpha r, y=\alpha r^{\prime}$, and then $\eta=(\gamma / z)$ to obtain equation (16) from equation (15).

Let us consider the first term in the curly brackets in eq. (16). Interchanging the order of integration, we obtain

$$
\begin{aligned}
d_{1}(\ell) & =\int_{0}^{1} \eta^{2 \ell+1}\left\{\int_{0}^{\infty} z \exp (-z) \exp (-z \eta) d z\right\} d \eta \\
& =\int_{0}^{1} \eta^{2 \ell+1}(1+\eta)^{-2} d \eta .
\end{aligned}
$$

We now examine the second term in the curly brackets of equation (16), namely,

$$
d_{2}(\ell)=\int_{0}^{\infty} z \exp (-z)\left(\int_{1}^{\infty} \eta^{-2 \ell-1} \exp (-z \eta) d \eta\right) d z
$$

Interchanging the order of integration in equation (18), we write the second term

$$
d_{2}(\ell)=\int_{1}^{\infty} \eta^{-2 \ell-1}(1+\eta)^{-2} d \eta
$$

By making the substitution $\eta=(1 / x)$, it follows that $d_{2}(\ell)=d_{1}(\ell)$.

From the above, we obtain the Schwinger upper limit for the total number of bound states associated with the Yukawa potential

$$
I_{s}[V]=(C / \alpha)^{2} \sum_{\ell=0}^{\infty} 2(2 \ell+1)^{-1} d_{1}(\ell)
$$

Interchanging the order of integration and summation in eq. (20),

$$
I_{S}[V]=(C / \alpha)^{2} \int_{0}^{1}(1-\eta)^{-2} \sum_{\ell=0}^{\infty} 2 \eta^{2 \ell+1}(2 \ell+1)^{-1}
$$

and observing that when $\eta<1$,

$$
\ln \left(\frac{1+\eta}{1-\eta}\right)=\sum_{\ell=0}^{\infty} \frac{2 \eta^{2 \ell+1}}{(2 \ell+1)},
$$

we write eq. (20) in the form

$$
I_{s}[V]=(C / \alpha)^{2} \int_{0}^{1} \frac{\ln \{(1+\eta) /(1-\eta)\}}{(1+\eta)^{2}} d \eta
$$

We show in Appendix A that the integral in eq. (21) equals $1 / 2$. Hence, our final result is

$$
I_{S}[V]=\frac{1}{2}\left\{I_{B}[V]\right\}^{2} \text {. }
$$

\section{Lieb Limits and Sobolev Inequality}

In three dimensions, Simon [10] gives a bound for large $(C / \alpha)$ due to Lieb of the form

$$
N \leq a_{3} \int|V(r)|^{3 / 2} d^{3} r \equiv I_{L}[V],
$$

where $a_{3} \leq 0.116$. For the potential (2), the Lieb limit $I_{L}[V]$ becomes

$$
I_{L}[V]=0.116 \times 6.062\left\{I_{B}[V]\right\}^{3 / 2}
$$

When $(C / \alpha)$ is small enough, bound states do not occur. The Sobolev inequality $[10,11]$ predicts that bound states do not occur whenever $I_{s I}[V]<1$, or equivalently, whenever 


$$
1>0.078 \times 6.062\left\{I_{B}[V]\right\}^{3 / 2} \equiv I_{S I}[V]
$$

That is, according to the Sobolev inequality, $N=0$ whenever $(C / \alpha)<1.65$

\section{Conclusions}

We have shown in sections 3,4 , and 5 that the upper limit for the total number $N$ of bound states associated with the Yukawa potential is for the Bargmann limit, eq. (9),

$$
N<\frac{1}{2} I_{B}[V]\left\{I_{B}[V]+1\right\}=N_{B},
$$

for the Schwinger limit,

$$
N<\frac{1}{2}\left\{I_{B}[V]\right\}^{2}=N_{S}
$$

and for the Lieb limit

$$
N<0.7032\left\{I_{B}[V]\right\}^{3 / 2}=N_{L} .
$$

Because $0<N_{s}[V]<N_{B}[V]$ for all $I_{B}[V]>0$, we conclude that the Schwinger upper limit, $N_{s}[V]$, is a more restrictive limit than $N_{B}[V]$. However, for large enough values of $I_{B}[V]$, the fractional difference $\left[\left(N_{B}\right.\right.$ - $\left.\left.N_{S}\right) / N_{B}\right]$, between the Schwinger and Bargmann upper bounds approaches zero as $\left\{1 / I_{B}[V]\right\}$ approaches zero.

When $(C / \alpha)>1.98, N_{L}<N_{s}$ and the Lieb limit is best for large $(C / \alpha)$ and for many bound states. When $(C / \alpha)<1.98, N_{s}<N_{L}$ and the Schwinger limit is best for the existence of only one bound state and for the approach to no binding, i.e., $1.98>(C / \alpha)>1.65$, and finally, the Sobolev inequality gives $N=0$ whenever $(C / \alpha)<1.65$. This value of 1.65 agrees to within 2 percent of the value $(C / \alpha)<1.68$ which Bonch-Bruevich and Glasko [5] determined numerically to give no bound state.

And finally, we observe from table 1 that even the Lieb limit is more than a factor of 3 greater than the numerically determined number of bound states given in reference 5 for values of $(C / \alpha)>8.92 .^{2}$

TABLE 1. Comparison of the Number of Bound States $N$ Determined

Numerically by Bonch-Bruevich and Glasko and the Three Upper

Limits of Bargmann [Eq. (9)], Schwinger [Eq. (22)], and Lieb

[Eq. (24)]. The Sobolev inequality is given by Eq. (25). All quantities are dimensionless.

\begin{tabular}{cccccc}
\hline$(\mathrm{C} / \alpha)$ & $\mathrm{N}$ & $\begin{array}{c}\text { Bargmann } \\
\text { Limit }\end{array}$ & $\begin{array}{c}\text { Schwinger } \\
\text { Limit }\end{array}$ & $\begin{array}{c}\text { Lieb } \\
\text { Limit }\end{array}$ & $\begin{array}{c}\text { Sobolev } \\
\text { Inequality }\end{array}$ \\
\hline 1.65 & 1 & 2.19 & 1.36 & 1.49 & 1.00 \\
1.68 & 1 & 2.25 & 1.41 & 1.53 & \\
1.90 & 1 & 2.76 & 1.81 & 1.84 & \\
1.98 & 1 & 2.95 & 1.96 & 1.96 & \\
2.00 & 1 & 3.00 & 2.00 & 1.99 & \\
6.00 & 1 & 21.00 & 18.00 & 10.33 & \\
6.45 & 2 & 24.03 & 20.80 & 11.52 & \\
7.00 & 2 & 28.00 & 24.50 & 13.02 & \\
8.50 & 2 & 40.38 & 36.13 & 17.43 & \\
8.92 & 5 & 44.24 & 39.78 & 18.73 & \\
\hline
\end{tabular}

- Reference 5.

\footnotetext{
The relative strengths of the Lieb, Schwinger, and Bargmann limits are depend. ent upon the potential $V(r)$. For example, the table analogous to table 1 for the attractive three-dimensional square well shows that the Bargmann limit is best in the range $2.47<V_{0 a^{2}}<10.74$ and the Lieb limit is best when $V_{0} a^{3}>10.74$, where $V_{0}$ is the
}

depth of the well and a is the width of the well. The Sobolev limit states that bound states do not occur when $V_{a}<2.11$ whereas the exact numerical result states that bound states do not occur when $V_{0 a^{2}}<2.47$. 
The author thanks Arnold Kahn and Jeremiah Lowney for their helpful discussions.

\section{References}

[1] Margenau, H; Lewis, M. Structure of spectral lines from plasmas, Rev. Mod. Phys. 31, 569 (1959).

[2] Hulthen, L.; Laurikainen, K.V. Approximate eigensolutions of $\left(d^{2} \varnothing / d x^{2}\right)+[a+b(\exp (-x) / x\}]=0$, Rev. Mod. Phys. 23, $1(1951)$.

[3] Stoneham, A.H. Theory of defects in solids Chapter 9, (Oxford: Clarendon Press, 1975).

[4] Toyozawa, Y. et al. On the interaction of additive electrons with the polarization in ionic crystals, II, Prog. Theor. Phys. 10, 57 (1953).

[5] Bonch-Bruevich, V.L.; Glasko, V.B. Energy levels in a Debye field, Opt. Spectrosc. 14, 264 (1963).

[6] Mott, N.F.; Gurney, R.W. Electronic processes in ionic crystals, chapter 3, (0xford: Clarendon Press, 1978).

[7] Lowney, J.; Kahn, A.K.; Blue, J.; Wilson, C., Disappearance of impurity levels in silicon and germanium due to screening, J. Appl. Phys. (to be published).

[8] Bargmann, V., On the number of bound states in a central field of force, Proc. Natl. Acad. Sci. USA, 47, 122 (1961).

[9] Schwinger, J., On the bound states of a given potential, Proc. Natl. Acad. Sci. USA, 47, 122 (1961).

[10] Simon, B. Functional integration and quantum physics, pp 88-97, (New York: Academic Press, 1979).

[11] Rosen, G., Minimum valve for $c$ in the Sobolev inequality, SIAMJ Appl. Math. 21, 30 (1971).

\section{Appendix}

In this appendix, we outline the evaluation of the integral that appears in eq. (21); namely,

$$
\left.I_{21}=\int_{0}^{1}(1+\eta)^{-2} \ln \{1+\eta) /(1-\eta)\right\} d \eta \text {. }
$$

We let $1+\eta=w$ and write

$$
I_{21}=\int_{1}^{2} w^{-2}\{\ln (w)-\ln (2-w)\} d w
$$

Referring to integral tables, we find that

$$
I_{21}=\frac{1}{2}+\operatorname{Lim}_{w \rightarrow 2} \frac{(2-w)}{4} \ln (2-w)=\frac{1}{2} .
$$

